\title{
Leucocyte telomere length, genetic variants at the TERT gene region and risk of pancreatic cancer
}

\author{
Ying Bao, ${ }^{1}$ Jennifer Prescott, ${ }^{1}$ Chen Yuan, ${ }^{2,3}$ Mingfeng Zhang, ${ }^{4}$ Peter Kraft, ${ }^{2,5}$ \\ Ana Babic, ${ }^{3}$ Vicente Morales-Oyarvide, ${ }^{3}$ Zhi Rong Qian, ${ }^{3}$ Julie E Buring, ${ }^{6,7}$ \\ Barbara B Cochrane, ${ }^{8}$ J Michael Gaziano, ${ }^{6,9}$ Edward L Giovannucci, ${ }^{1,2,10}$

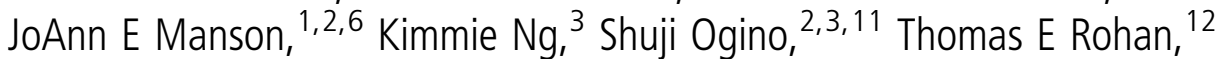 \\ Howard D Sesso, ${ }^{2,6}$ Meir J Stampfer, ${ }^{1,2,10}$ Charles S Fuchs, ${ }^{1,3}$ Immaculata De Vivo, ${ }^{1,2}$ \\ Laufey T Amundadottir, ${ }^{4}$ Brian M Wolpin ${ }^{3,13}$
}

\begin{abstract}
- Additional material is published online only. To view please visit the journal online (http://dx.doi.org/10.1136/ gutjnl-2016-312510).

For numbered affiliations see end of article.
\end{abstract}

\section{Correspondence to}

Ying Bao, Channing Laboratory, Department of Medicine, Brigham and Women's Hospital and Harvard Medical School 181 Longwood Avenue, Boston, MA 02115, USA; ying.bao@ channing.harvard.edu

Received 27 June 2016 Revised 22 August 2016 Accepted 1 October 2016 Published Online First 21 October 2016

\section{SLinked}

- http://dx.doi.org/10.1136 gutjnl-2016-313156 CrossMark

To cite: Bao Y, Prescott J, Yuan C, et al. Gut

2017:66:1116-1122.

\section{ABSTRACT}

Objective Telomere shortening occurs as an early event in pancreatic tumorigenesis, and genetic variants at the telomerase reverse transcriptase (TERT) gene region have been associated with pancreatic cancer risk. However, it is unknown whether prediagnostic leucocyte telomere length is associated with subsequent risk of pancreatic cancer.

Design We measured prediagnostic leucocyte telomere length in 386 pancreatic cancer cases and 896 matched controls from five prospective US cohorts. ORs and $95 \%$ Cls were calculated using conditional logistic regression. Matching factors included year of birth, cohort (which also matches on sex), smoking status, fasting status and month/year of blood collection. We additionally examined single-nucleotide polymorphisms (SNPs) at the $T E R T$ region in relation to pancreatic cancer risk and leucocyte telomere length using logistic and linear regression, respectively.

Results Shorter prediagnostic leucocyte telomere length was associated with higher risk of pancreatic cancer (comparing extreme quintiles of telomere length, OR $1.72 ; 95 \% \mathrm{Cl} 1.07$ to $2.78 ; p_{\text {trend }}=0.048$ ). Results remained unchanged after adjustment for diabetes, body mass index and physical activity. Three SNPs at TERT (linkage disequilibrium $r^{2}<0.25$ ) were associated with pancreatic cancer risk, including rs401681 (per minor allele OR 1.33; 95\% Cl 1.12 to $1.59 ; p=0.002$ ), rs 2736100 (per minor allele OR 1.36; $95 \% \mathrm{Cl} 1.13$ to 1.63; $p=0.001$ ) and rs2736098 (per minor allele OR $0.75 ; 95 \% \mathrm{Cl} 0.63$ to $0.90 ; p=0.002$ ). The minor allele for rs401681 was associated with shorter telomere length ( $p=0.023$ ).

Conclusions Prediagnostic leucocyte telomere length and genetic variants at the TERT gene region were associated with risk of pancreatic cancer.

\section{INTRODUCTION}

Pancreatic cancer is the third leading cause of cancer death in the USA. ${ }^{1}$ The large number of deaths from pancreatic cancer is due in part to late diagnosis, with $>80 \%$ of patients presenting with advanced, incurable disease. ${ }^{2}$ To facilitate early detection, it is critical to identify those individuals at increased risk. ${ }^{3}$ Nevertheless, known

\section{Significance of this study}

What is already known on this subject?

- Telomere shortening occurs as an early event in pancreatic tumorigenesis, and genetic variants at the telomerase reverse transcriptase (TERT) gene region have been associated with pancreatic cancer risk.

- However, the mechanisms by which common variants at the TERT gene region impact development of sporadic pancreatic cancer are unknown, and the role of leucocyte telomere length as a predictive marker for pancreatic cancer has not been extensively studied.

\section{What are the new findings?}

- We found that shorter prediagnostic leucocyte telomere length was associated with increased risk of pancreatic cancer.

- Three single-nucleotide polymorphisms at the TERT gene region were associated with pancreatic cancer risk, including rs401681, rs2736100 and rs2736098.

- Interestingly, the minor allele for rs401681 was associated with shorter telomere length and increased risk of pancreatic cancer.

How might it impact on clinical practice in the foreseeable future?

- The findings from this study, along with data from previous tumour tissue and genetic studies, strongly implicate telomere maintenance in the development of pancreatic cancer.

- These findings strongly support further investigation of non-invasive testing of telomere biology as a component of pancreatic cancer risk stratification strategies.

predisposing factors for pancreatic cancer, including obesity, diabetes, smoking and chronic pancreatitis, are inadequate to meaningfully risk stratify the general population. ${ }^{4}$

Telomeres are DNA repeats at the ends of chromosomes with a critical role in maintaining 
genomic stability. ${ }^{5}$ Human telomeres shorten with each cell division, and the rate of shortening across individuals is influenced by genetic and environmental factors. ${ }^{6}{ }^{7}$ Interestingly, telomere shortening occurs as an early event in pancreatic tumorigenesis. ${ }^{8} 9$ The telomerase reverse transcriptase (TERT) gene at chromosome $5 \mathrm{p} 15.33$ encodes the catalytic subunit of telomerase reverse transcriptase, a component of the protein and RNA complex that maintains telomere ends. Recent genome-wide association studies (GWAS) have identified inherited genetic variation at the TERT gene region associated with risk of developing pancreatic cancer ${ }^{10} 11$ and several other malignancies. ${ }^{12} 13$ Nevertheless, the mechanisms by which common variants at the TERT gene region impact development of sporadic pancreatic cancer are unknown, and the role of leucocyte telomere length as a predictive marker for pancreatic cancer has not been extensively studied. ${ }^{14} 15$

We examined prediagnostic leucocyte telomere length and subsequent risk of pancreatic cancer in five prospective US cohorts with up to 26 years of follow-up since blood collection. We additionally investigated single-nucleotide polymorphisms (SNPs) at the TERT gene region and their relationship to leucocyte telomere length and pancreatic cancer risk.

\section{METHODS}

\section{Study participants}

Pancreatic cancer cases and controls were included from five US prospective cohorts. Briefly, the Nurses' Health Study (NHS) enrolled 121700 female nurses aged 30-55 years in 1976. The Health Professionals Follow-up Study (HPFS) enrolled 51529 male health professionals aged 40-75 years in 1986. The Physicians' Health Study I (PHS) is a randomised clinical trial of aspirin and $\beta$-carotene and enrolled 22071 healthy male physicians aged 40-84 years in 1982. After the randomised trial ended, participants were followed as an observational cohort. The Women's Health Initiative Observational Study (WHI-OS) enrolled 93676 postmenopausal women aged 50-79 years between 1994 and 1998. The Women's Health Study (WHS) is a randomised clinical trial of low-dose aspirin and vitamin $\mathrm{E}$ and enrolled 39876 healthy female health professionals aged $\geq 45$ years between 1992 and 1995 . The trial was completed in 2004, and participants were followed as an observational cohort.

Blood samples were collected from 32826 women in NHS from 1989 to 1990, 18225 men in HPFS from 1993 to 1995, 14916 men in PHS from 1982 to 1984, 93676 women in WHI-OS from 1994 to 1998 and 28345 women in WHS from 1992 to 1995 . Individual characteristics and habits were obtained from baseline questionnaires in PHS, WHI-OS and WHS and from questionnaires preceding blood draw in NHS and HPFS.

\section{Pancreatic cancer cases and controls}

We included pancreatic adenocarcinoma cases diagnosed through 2008 with available blood samples and no history of cancer, except non-melanoma skin cancer. Incident cases were identified by self-report or during follow-up of a participant's death. Medical records of the cases were reviewed by study physicians blinded to exposure data. Eligible controls were cohort participants who provided a blood sample and were alive and free of cancer at the date of the case's diagnosis. We randomly selected up to three controls for each case, matching on year of birth, prospective cohort (which matched on sex), smoking status (never, past, current), fasting status at blood collection ( $<8$ hours, $\geq 8$ hours) and month/year of blood collection.
The nested case-control set consisted of 472 pancreatic cancer cases and 1071 controls. Because telomere dynamics may differ across races/ethnicities ${ }^{16}{ }^{17}$ and SNPs at the TERT gene region were identified in individuals of European descent, ${ }^{10} 11$ Caucasian subjects were excluded, resulting in 456 cases and 1035 matched controls. To minimise the influence of subclinical malignancy on leucocyte telomere length, we excluded casecontrol sets in which the pancreatic cancer case was diagnosed within two years of blood collection, resulting in 386 pancreatic cancer cases and 896 matched controls for analysis.

\section{Prediagnostic leucocyte telomere length measurement}

Genomic DNA was extracted from peripheral blood leucocytes using QIAmp (Qiagen, Chatsworth, California, USA) 96-spin blood protocol. PicoGreen quantitation of genomic DNA was performed using Molecular Devices 96-well spectrophotometer. The ratio of telomere repeat copy number to a single-gene copy number $(\mathrm{T} / \mathrm{S})$ was determined by modified, high-throughput ${ }^{18}$ quantitative real-time PCR telomere assay ${ }^{19}$ run Applied Biosystems 7900HT Sequence Detection System (Foster City, California, USA). Triplicate reactions were performed. Leucocyte relative telomere length is reported as exponentiated sample T/S ratio corrected for a reference sample. Telomere and single-gene assay coefficients of variation (CVs) for triplicates were $0.6 \%$ and $0.5 \%$, respectively. CVs for the exponentiated $\mathrm{T} / \mathrm{S}$ ratio were $12.9 \%$.

\section{SNP selection and genotyping}

We genotyped four SNPs at the TERT gene region previously associated with cancer risk, including rs401681 (pancreatic cancer) ${ }^{11}$ rs2736100 (glioma and lung cancer), ${ }^{20}{ }^{21}$ rs402710 (lung cancer) ${ }^{20}$ and rs2853676 (glioma). ${ }^{21}$ DNA was extracted centrally from archived buffy coat samples with QIAGEN QIAmp and whole genome amplified with GE Healthcare Genomiphi. All genotyping was performed at Partners HealthCare Center for Personalized Genetic Medicine using a custom-designed Illumina Golden Gate genotyping assay. Replicate samples included for quality control $(\mathrm{N}=44$ sample groups) had mean genotype concordance of $97.2 \%$ across the four SNPs. No SNPs deviated from Hardy-Weinberg equilibrium at $\mathrm{p}<0.01$.

In addition, 644 pancreatic cancer cases and 1171 controls from the five cohorts were included in recent GWAS of pancreatic cancer (PanScan studies). ${ }^{10} 1122$ From these studies, we obtained SNP genotypes for our cohort participants to mark the six independent risk loci at TERT identified in a recent, large, multicancer analysis. ${ }^{23}$ Genotyping and imputation methods in the PanScan studies have been described previously. ${ }^{10}$ Among these subjects, 290 cases and 232 controls were included in the nested case-control study of telomere length. Online supplementary table 1 summarises the numbers of cases and controls with leucocyte telomere length, locally genotyped SNPs and SNPs genotyped in PanScan.

\section{Statistical analysis}

To standardise telomere length values, we calculated z-scores (SDs from the mean) of log-transformed telomere length within each cohort. Participants were categorised into quintiles based on the z-score distributions among all controls. To compute ORs and 95\% CIs, we used conditional logistic regression. In multivariable models, we adjusted for history of diabetes (yes, no), body mass index (BMI, <18.5, 18.5-24.9, 25-29.9, $\geq 30 \mathrm{~kg} / \mathrm{m}^{2}$ ) and physical activity (quartiles). $\mathrm{p}$ Values for trend were calculated by the Wald test of a score variable that 
contained median values of quintiles. Because two previous studies suggested a non-linear relation between telomere length and pancreatic cancer risk, ${ }^{14}{ }^{24}$ we tested non-linearity using restricted cubic spline regression. ${ }^{25}$ We conducted stratified analyses by sex, age at blood draw ( $<63, \geq 63$ years; median), smoking status (never, ever) and time between blood collection and cancer diagnosis $(<6.7, \geq 6.7$ years; median). Tests for interaction were performed by the Wald test of cross-product terms.

Associations between SNPs at TERT and pancreatic cancer risk were examined by assigning a value of 0,1 or 2 according to a participant's number of minor alleles and including this genotype as a continuous variable (additive model) in logistic regression models. For analysis of SNPs in the nested casecontrol study, conditional logistic regression models were adjusted for the aforementioned covariates. For analyses of SNPs using PanScan genotypes in which no case-control matching was present, unconditional logistic regression models were adjusted for age and cohort. The association of SNPs with telomere length was assessed using linear regression, with models adjusted for age, case-control status, prospective cohort (also adjusts for sex), smoking status, history of diabetes, BMI and physical activity using an additive genetic model. All analyses were performed with SAS V.9.3 statistical package. All p values were two-sided.

\section{RESULTS}

The median time from collection of DNA to cancer diagnosis was 6.7 years in cases. As expected, individuals with shorter telomere length were older and more likely to be current smokers (table 1, characteristics among controls). The Spearman correlation coefficient between leucocyte telomere length and age was $-0.14 \quad(\mathrm{p}<0.0001)$ among controls. Online supplementary table 2 shows the characteristics of pancreatic cancer cases and matched controls in each cohort.

We observed a statistically significant inverse association between prediagnostic leucocyte telomere length and risk of pancreatic cancer (table 2). Compared with the highest quintile of telomere length z-score, individuals in quintiles four to one had multivariable ORs (95\% CIs) of 1.35 (0.90 to 2.04), 1.23 (0.79 to 1.93$), 1.27$ (0.79 to 2.02 ) and 1.72 (1.07 to 2.78$)$, respectively $\left(\mathrm{p}_{\text {trend }}=0.048\right)$. Adjustment for history of diabetes, BMI and physical activity did not materially alter the results (table 2).
Restricted cubic spline curve analysis was consistent with a linear relationship of telomere length and pancreatic cancer risk ( $p_{\text {nonlinearity }}>0.05$ ). No statistically significant heterogeneity was observed across the five cohorts ( $p_{\text {heterogeneity }}=0.24$ ). No statistically significant interaction was observed by sex, age at blood collection, smoking status or time between blood collection and cancer diagnosis (all $p_{\text {interaction }} \geq 0.32$ ).

We genotyped four SNPs at the TERT gene region, including rs401681 that was associated with pancreatic cancer risk in the PanScan II GWAS ${ }^{11}$ and three SNPs identified in GWAS of lung cancer and glioma, ${ }^{20}{ }^{21}$ rs2736100, rs402710 and rs2853676. Two of these SNPs, rs401681 and rs2736100, were associated with pancreatic cancer risk in our nested case-control study at a Bonferroni-corrected $\mathrm{p}$ value threshold of 0.0125 (0.05/4 SNPs) (table 3). The per minor allele ORs were 1.33 (95\% CI 1.12 to 1.59) for rs401681 and 1.36 (95\% CI 1.13 to 1.63) for rs2736100. The PanScan III study identified a second independent locus at TERT marked by rs $2736098,{ }^{10}$ and further studies have suggested multiple independent risk loci for cancer in this genomic region. ${ }^{23} 262728$ Based on the six independent risk loci identified at TERT in a large multicancer analysis by Wang et $a l,{ }^{23}$ we obtained PanScan genotypes marking these loci for cases and controls from our five cohorts. An SNP at one of the six loci was associated with pancreatic cancer risk to $\mathrm{p}<0.008$ (0.05/6 SNPs), rs2736098 (OR 0.75 ; 95\% CI 0.63 to 0.90 ; table 4). A second SNP was nominally significant, rs451360 (OR 1.20; 95\% CI 1.00 to 1.43 ); this SNP is known from finemapping analyses to mark the same risk locus as rs $401681 .^{23}$

We next evaluated the association of the three SNPs related to pancreatic cancer risk with leucocyte telomere length (table 5). rs401681 was statistically significantly associated with leucocyte telomere length in linear regression models $(p=0.023)$, although power was limited for rs2736098 due to modest overlap between the nested case-control and PanScan populations and lower minor allele frequency. For rs401681, the minor allele was associated with both shorter telomere length (table 5) and increased risk of pancreatic cancer (table 3).

\section{DISCUSSION}

In this large prospective study of five US cohorts, shorter prediagnostic leucocyte telomere length was associated with higher risk of developing pancreatic cancer, independent of known

Table 1 Characteristics by quintile of leucocyte telomere length among controls in five prospective cohorts

\begin{tabular}{|c|c|c|c|c|c|}
\hline \multirow[b]{2}{*}{ Characteristic } & \multicolumn{5}{|c|}{ Quintiles of leucocyte telomere length, z-score } \\
\hline & Q5 (longest) & Q4 & Q3 & Q2 & Q1 (shortest) \\
\hline No. of controls & 179 & 180 & 179 & 178 & 180 \\
\hline Age, years, mean (SD) & $61.1(8.3)$ & $61.1(8.5)$ & $61.9(9.1)$ & $63.8(9.2)$ & $64.1(8.6)$ \\
\hline Men, \% & 35.8 & 35.2 & 35.7 & 35.2 & 34.6 \\
\hline Height, inches, mean (SD) & $66.5(3.5)$ & $66.2(3.8)$ & $66.7(3.9)$ & $66.5(3.7)$ & $66.7(3.8)$ \\
\hline Body mass index, $\mathrm{kg} / \mathrm{m}^{2}$, mean (SD) & $25.7(4.8)$ & $26.1(4.6)$ & $25.6(4.2)$ & $25.1(3.7)$ & $25.6(4.0)$ \\
\hline Physical activity, MET-hour/week, mean (SD) & $20.5(21.3)$ & $21.5(23.9)$ & $20.2(26.3)$ & $20.5(29.4)$ & $18.0(22.6)$ \\
\hline \multicolumn{6}{|l|}{ Smoking status, \% } \\
\hline Never & 43 & 49.6 & 42.1 & 40.3 & 42.2 \\
\hline Past & 43.6 & 37.1 & 44.7 & 47.2 & 39 \\
\hline Current & 13.4 & 13.3 & 13.2 & 12.5 & 18.8 \\
\hline History of diabetes mellitus, \% & 4.4 & 4.2 & 2.2 & 1.1 & 5.4 \\
\hline Regular multivitamin use, \% & 43 & 41.8 & 45.2 & 32.2 & 38.4 \\
\hline Alcohol ( $\geq 1$ drink/day), $\%$ & 22.4 & 22.8 & 23.4 & 19.3 & 23.6 \\
\hline
\end{tabular}


Table 2 Association between prediagnostic leucocyte telomere length and future risk of pancreatic cancer

\begin{tabular}{|c|c|c|c|c|c|c|}
\hline & \multicolumn{5}{|c|}{ Quintiles of leucocyte telomere length, z score } & \multirow[b]{2}{*}{ p Value, trend* } \\
\hline & Q5 (longest) & Q4 & Q3 & Q2 & Q1 (shortest) & \\
\hline No. of cases & 64 & 84 & 72 & 71 & 95 & \\
\hline No. of controls & 179 & 180 & 179 & 178 & 180 & \\
\hline OR $(95 \% \mathrm{Cl}) \dagger$ & 1.0 & $1.35(0.90-2.04)$ & $1.23(0.79-1.93)$ & $1.27(0.79-2.02)$ & $1.72(1.07-2.78)$ & 0.048 \\
\hline OR $(95 \%$ Cl) $\ddagger$ & 1.0 & $1.29(0.85-1.96)$ & $1.20(0.76-1.89)$ & $1.27(0.80-2.04)$ & $1.67(1.03-2.72)$ & 0.056 \\
\hline
\end{tabular}

${ }^{*} p$ Value for trend calculated by the Wald test of a score variable containing median values of quintiles.

tConditional logistic regression model conditioned on matching factors: year of birth, prospective cohort (HPFS, NHS, PHS, WHI-OS, WHS, which also matches on sex), smoking status (never, past, current), fasting status at blood collection ( $<8$ hours, $\geq 8$ hours) and month/year of blood collection.

$\neq$ Conditional logistic regression model conditioned on matching factors and adjusted for history of diabetes mellitus (yes, no), body mass index $\left(<18.5,18.5-24.9,25-29.9, \geq 30 \mathrm{~kg} / \mathrm{m}^{2}\right)$ and physical activity (quartiles).

HPFS, Health Professionals Follow-up Study; NHS, Nurses' Health Study; PHS, Physicians' Health Study I; WHI-OS, Women's Health Initiative Observational Study; WHS, Women's Health Study.

predisposing factors. Three SNPs at the TERT gene region were also associated with pancreatic cancer risk, including two SNPs identified in the PanScan GWAS. ${ }^{10}{ }^{11}$ In our five cohorts, the minor allele of rs401681 was associated with both shorter leucocyte telomere length and higher risk of pancreatic cancer. In aggregate, these data implicate telomere maintenance in the development of pancreatic cancer. Furthermore, they support further investigation of non-invasive testing of telomere biology as a component of pancreatic cancer risk stratification strategies.

Large studies and meta-analyses have assessed the association of peripheral leucocyte telomere length and overall cancer risk, with inconsistent results. ${ }^{29} 303132$ Initial studies suggested that shorter telomere length was associated with increased cancer risk and mortality, while others have suggested only an association of shorter telomere length with worse cancer survival. ${ }^{32} 33$ These inconsistencies may result from differences in study design and assay performance and the potential impact of confounding factors. ${ }^{34}$ The kinetics of telomere loss appear to vary by timing of measurement before cancer diagnosis ${ }^{35}$ and treatment after diagnosis, ${ }^{36}$ impairing the ability to combine data from studies with different designs. In the current study, all blood samples were collected prospectively $>2$ years prior to diagnosis and before any cancer-directed treatments, limiting the impact of these factors on our results. Pooling data across studies and laboratories can be complex due to methodological differences in measuring telomere length. ${ }^{37}$ In the current study, all samples were analysed together in the same laboratory using the same analytic protocol. Confounding by exposures such as smoking and obesity may affect associations. We included multiple potential confounding factors in our multivariable-adjusted models, noting little change in our results. Notably, recent studies have constructed genetic scores based on the number of inherited telomere-shortening alleles and noted associations with cancer risk, suggesting an independent effect of telomere length on cancer risk. 38394041

Previous smaller studies focused on leucocyte telomere length and pancreatic cancer risk have yielded inconsistent results. In a hospital-based case-control study, shorter telomere length was associated with higher risk for pancreatic cancer across most of the distribution of length. ${ }^{24}$ However, the temporality of this relationship could not be established due to the retrospective study design, with blood samples collected at the time of cancer diagnosis. A prospective study of 193 pancreatic cancer cases in the Alpha-Tocopherol, Beta-Carotene Cancer Prevention study found that longer telomere length was associated with higher pancreatic cancer risk among individuals diagnosed within the first five years of blood collection. ${ }^{15}$ However, this study was conducted among male smokers in Finland, limiting generalisability of the results. A prospective study in the European Prospective Investigation into Cancer and Nutrition suggested no overall association of leucocyte telomere length and pancreatic cancer risk. $^{14}$ Secondary analyses suggested modest increases in risk for individuals with both short and long telomere length.

Telomere shortening is known to be present at the early stages of pancreatic tumorigenesis. ${ }^{9}{ }^{42}$ When telomeres shorten to a critical length, the DNA damage response triggers senescence or apoptosis. ${ }^{5}$ In preneoplastic cells, if protective mechanisms such as the p53 tumour suppressor are inactive, the cell continues to proliferate and telomeres become extremely short,

Table 3 Association of genotyped single-nucleotide polymorphisms (SNPs) at the TERT gene region with pancreatic cancer risk in five prospective cohorts

\begin{tabular}{|c|c|c|c|c|c|c|c|c|c|}
\hline \multirow[b]{2}{*}{ SNP } & \multirow{2}{*}{$\begin{array}{l}\mathrm{LDt} \\
\mathrm{r}^{2}\end{array}$} & \multirow[b]{2}{*}{ Major allele } & \multirow[b]{2}{*}{ Minor allele } & \multicolumn{2}{|c|}{ Participants } & \multicolumn{2}{|l|}{ MAF } & \multirow{2}{*}{$\begin{array}{l}\text { Per minor allele } \\
\text { OR }(95 \% \mathrm{Cl})^{*}\end{array}$} & \multirow[b]{2}{*}{$p$ Value } \\
\hline & & & & Cases & Controls & Cases & Controls & & \\
\hline rs401681 & - & G & A & 362 & 846 & 0.47 & 0.40 & $1.33(1.12-1.59)$ & 0.002 \\
\hline rs 2736100 & 0.01 & C & A & 363 & 845 & 0.55 & 0.48 & $1.36(1.13-1.63)$ & 0.001 \\
\hline rs402710 & 0.59 & G & $A$ & 360 & 847 & 0.37 & 0.33 & $1.20(1.00-1.44)$ & 0.047 \\
\hline rs2853676 & 0.007 & G & A & 363 & 841 & 0.26 & 0.27 & $0.94(0.76-1.15)$ & 0.540 \\
\hline
\end{tabular}

* Conditional logistic regression model conditioned on matching factors (year of birth, prospective cohort, smoking status, fasting status and month/year of blood draw) and adjusted for history of diabetes mellitus (yes, no), body mass index $\left(<18.5,18.5-24.9,25-29.9, \geq 30 \mathrm{~kg} / \mathrm{m}^{2}\right)$ and physical activity (quartiles). OR reflects the OR per minor allele in an additive genetic model.

TLD of SNPs with rs401681 based on 1000G CEU data.

$L D$, linkage disequilibrium; MAF, minor allele frequency; TERT, telomerase reverse transcriptase. 
Table 4 Association of single-nucleotide polymorphisms (SNPs) marking six previously identified, independent risk loci at the TERT gene region with pancreatic cancer in five prospective cohorts

\begin{tabular}{|c|c|c|c|c|c|c|c|c|c|}
\hline \multirow[b]{2}{*}{ Risk region } & \multirow[b]{2}{*}{ SNP } & \multirow[b]{2}{*}{ Major allele } & \multirow[b]{2}{*}{ Minor allele } & \multicolumn{2}{|c|}{ Participants } & \multicolumn{2}{|l|}{ MAF } & \multirow[b]{2}{*}{ Per minor allele OR $(95 \% \mathrm{CI})^{*}$} & \multirow[b]{2}{*}{ p Value } \\
\hline & & & & Cases & Controls & Cases & Controls & & \\
\hline 1 & rs7726159 & $\mathrm{C}$ & $A$ & 644 & 1171 & 0.33 & 0.34 & $0.97(0.81-1.16)$ & 0.741 \\
\hline 2 & rs451360 & $\mathrm{C}$ & $A$ & 644 & 1171 & 0.24 & 0.22 & $1.20(1.00-1.43)$ & 0.044 \\
\hline 3 & rs2853677 & $A$ & G & 644 & 1171 & 0.40 & 0.41 & $0.92(0.79-1.08)$ & 0.320 \\
\hline 4 & rs2736098 & $\mathrm{C}$ & $\mathrm{T}$ & 644 & 1171 & 0.23 & 0.28 & $0.75(0.63-0.90)$ & 0.002 \\
\hline 5 & rs13172201 & $\mathrm{T}$ & $\mathrm{C}$ & 644 & 1171 & 0.26 & 0.26 & $1.02(0.84-1.25)$ & 0.808 \\
\hline 6 & rs10069690 & $\mathrm{C}$ & $\mathrm{T}$ & 644 & 1171 & 0.23 & 0.24 & $1.00(0.83-1.21)$ & 0.996 \\
\hline
\end{tabular}

Table 5 Association of single-nucleotide polymorphisms (SNPs) at the TERT gene region with leucocyte telomere length in five prospective cohorts

\begin{tabular}{lllllll}
\hline SNP & Major allele & Minor allele & Subjects & $\boldsymbol{\beta}$-Value* & SE & $\boldsymbol{p}$ Value \\
\hline rs401681 & G & A & 1208 & -0.09 & 0.04 & 0.023 \\
rs2736100 & C & A & 1208 & -0.04 & 0.04 & 0.394 \\
rs2736098 & C & T & 522 & 0.05 & 0.08 & 0.519
\end{tabular}

* Linear regression model per SNP minor allele adjusted for age (continuous), casecontrol status, prospective cohort (HPFS, NHS, PHS, WHI-OS, WHS, also adjusts for sex), smoking status (never, past, current), history of diabetes mellitus (yes, no), body mass index $\left(<18.5,18.5-24.9,25-29.9, \geq 30 \mathrm{~kg} / \mathrm{m}^{2}\right)$ and physical activity (quartiles) using an additive genetic model.

HPFS, Health Professionals Follow-up Study; NHS, Nurses' Health Study; PHS,

Physicians' Health Study I; TERT, telomerase reverse transcriptase; WHI-OS, Women's Health Initiative Observational Study; WHS, Women's Health Study.

ultimately causing chromosomal instability and invasive malignancy. ${ }^{5}$ Thus, telomere length in tissues may be important in determining whether preneoplastic lesions progress to invasive disease. Whether telomere length measured in white blood cells reflects telomere length in the pancreas is unknown. However, prior studies have indicated that telomere shortening proceeds at similar rates across human somatic tissues ${ }^{43}$ and may be influenced by both genetic and environmental factors. ${ }^{6} 7$

The TERT gene encodes the catalytic subunit of telomerase reverse transcriptase, a component of the protein and RNA complex that maintains telomere ends. SNPs at chromosome $5 \mathrm{p} 15.33$, adjacent to or within the TERT gene, are associated with the development of multiple malignancies. ${ }^{26} 44$ Previously, the PanScan studies observed altered pancreatic cancer risk in association with two independent loci at the TERT gene region marked by rs401681 $1^{11}$ and rs $2736098 .^{10}$ In the present study, we observed similar associations of pancreatic cancer risk with these two SNPs and also noted an association of a third SNP (rs2736100) in low linkage disequilibrium with the others. Of these SNPs, rs401681 was also associated with leucocyte telomere length, such that the minor allele at rs401681 was associated with higher pancreatic cancer risk and shorter telomere length. Similarly, multiple independent variants have been identified at the TERT gene region related to breast and ovarian cancer risk, with a subset of these variants also associated with leucocyte telomere length. ${ }^{26}$ Similar to our results related to pancreatic cancer, the minor allele at the locus represented by rs2736098 was associated with longer telomeres and lower risk of oestrogen receptor-positive breast cancer. Furthermore, the A allele at rs2736100 was associated with shorter telomere length in a prior $\mathrm{GWAS}^{45}$ and with increased pancreatic cancer risk in the current study. In aggregate, these data suggest that SNPs at the TERT gene region might be associated with shorter telomere length and higher risk of pancreatic cancer.

Strengths of the present study include the prospective design, large sample size for studies of pancreatic cancer and long, nearly complete follow-up. The exclusion of cases diagnosed within two years of blood draw further minimised potential bias due to reverse causation. The multidimensional data from the five cohorts provided a unique opportunity to conduct integrative analyses of leucocyte telomere length, SNPs at the TERT gene region and pancreatic cancer risk. The extensive covariate data from these cohorts also allowed for rigorous control of potential confounding and evaluation of effect modification.

A limitation of our study was an inability to assess risk related to telomere attrition rate as we were only able to measure telomere length at one point in time. However, most prospective studies have evaluated outcomes related to a one-time telomere length measurement, and whether longitudinal measurement will better predict cancer risk is unknown. We cannot be certain that leucocyte telomere length reflects telomere length in pancreatic tissue. However, telomere length is highly synchronised in fetal tissues ${ }^{46}$ and among white blood cells, umbilical artery cells and skin cells at birth, ${ }^{17} 4748$ and interindividual variation in telomere length far exceeds variation between different tissues from the same individual. ${ }^{17} 48 \mathrm{~A}$ recent study also showed that the rate of telomere shortening was similar across leucocytes, skin, skeletal muscle and subcutaneous fat, ${ }^{43}$ suggesting that blood serves as an adequate proxy for peripheral tissues. Although the blood samples were collected from different cohorts, it is unlikely that differences in blood collection affected the quality of the genomic DNA because genomic DNA is coiled around histones and very stable when housed in intact nuclei of cells, even when frozen. Furthermore, the effect of potential differences in the handling of biological materials between studies would likely be mitigated because the T/S ratios from each study were normalised using z-scores, which ranks each participant in each study by telomere length based on their respective distributions. Finally, our study population consisted only of white participants, so we were not able to examine other ethnic groups.

In conclusion, in this large study of five prospective cohorts, we found that shorter leucocyte telomere length and genetic variants at the TERT gene locus were associated with higher subsequent risk of pancreatic cancer. Overall, these data support 
a role for telomere maintenance in the development of pancreatic cancer. Furthermore, they suggest that further investigation of non-invasive testing related to telomere biology may have potential as a component of pancreatic cancer risk stratification strategies.

\section{Author affiliations}

${ }^{1}$ Channing Division of Network Medicine, Department of Medicine, Brigham and Women's Hospital, and Harvard Medical School, Boston, Massachusetts, USA ${ }^{2}$ Department of Epidemiology, Harvard T.H. Chan School of Public Health, Boston, Massachusetts, USA

${ }^{3}$ Department of Medical Oncology, Dana-Farber Cancer Institute, Boston, Massachusetts, USA

${ }^{4}$ Laboratory of Translational Genomics, Division of Cancer Epidemiology and Genetics, National Cancer Institute, National Institutes of Health, Bethesda, Maryland, USA

${ }^{5}$ Department of Biostatistics, Harvard School of Public Health, Boston,

Massachusetts, USA

${ }^{6}$ Division of Preventive Medicine, Department of Medicine, Brigham and Women's Hospital and Harvard Medical School, Boston, Massachusetts, USA

${ }^{7}$ Department of Ambulatory Care and Prevention, Harvard Medical School, Boston, Massachusetts, USA

${ }^{8}$ University of Washington School of Nursing, Seattle, Washington, USA

${ }^{9}$ Massachusetts Veterans Epidemiology Research and Information Center (MAVERIC), VA Boston Healthcare System

${ }_{10}$ Department of Nutrition, Harvard School of Public Health, Boston, Massachusetts, USA

${ }^{11}$ Division of MPE Molecular Pathological Epidemiology, Department of Pathology, Brigham and Women's Hospital and Harvard Medical School, Boston, Massachusetts, USA

${ }^{12}$ Department of Epidemiology and Population Health, Albert Einstein College of Medicine, Bronx, New York, USA

${ }^{13}$ Department of Medicine, Brigham and Women's Hospital, and Harvard Medical School, Boston, Massachusetts, USA

Acknowledgements The authors thank the participants and staff of the Nurses' Health Study, Health Professionals Follow-up Study, Physicians' Health Study, Women's Health Initiative and Women's Health Study, for their contributions as wel as the following state cancer registries for their help. $A L, A Z, A R, C A, C O, C T, D E$, $F L, G A, I D, I L, I N, I A, K Y, L A, M E, M D, M A, M I, N E, N H, N J, N Y, N C, N D, O H, O K$, OR, PA, RI, SC, TN, TX, VA, WA, WY. The authors assume full responsibility for analyses and interpretation of these data.

Contributors YB had full access to all of the data in the study and takes responsibility for the integrity of the data and the accuracy of the data analysis. Study concept and design: YB, BMW. Acquisition of data: PK, JEB, BBC, JMG, JAEM, TER, HDS, MJS, CSF, IDV, LTA, BMW. Analysis and interpretation of data: YB, JP, CY, MZ, PK, AB, VM-O, ZRQ, JEB, BBC, JMG, ELG, JAEM, KN, SO, TER, HDS, MJS, CSF, IDV, LTA, BMW. Drafting of the manuscript: YB, BMW. Critical revision of the manuscript for important intellectual content: $\mathrm{YB}, \mathrm{JP}, \mathrm{CY}, \mathrm{MZ}, \mathrm{PK}, \mathrm{AB}, \mathrm{VM}-\mathrm{O}$, ZRQ, JEB, BBC, JMG, ELG, JAEM, KN, SO, TER, HDS, MJS, CSF, IDV, LTA, BMW. Statistical analysis: YB. Obtained funding: CF, BMW. Administrative, technical, or material support: JP, CY, MZ. Study supervision: BMW.

Funding NHS is supported by NIH grants UM1 CA186107, P01 CA87969 and R01 CA49449. HPFS is supported by NIH grant UM1 CA167552. PHS is supported by NIH grants CA 97193, CA 34944, CA 40360, HL 26490 and HL 34595. The WHI programme is funded by the NIH through contracts N01WH22110, 24152, 321002, 32105-6, 32108-9, 32111-13, 32115, 32118-32119, 32122, 42107-26, 42129-32, and 44221. WHS is supported by NIH grants CA047988, HL043851 and HL080467. Additional support from KL2/Catalyst Medical Research Investigator Training award (an appointed KL2 award) from Harvard Catalyst | The Harvard Clinical and Translational Science Center (National Center for Research Resources and the National Center for Advancing Translational Sciences, NIH KL2 TR001100) to YB; from the Robert T. and Judith B. Hale Fund for Pancreatic Cancer, Perry S. Levy Fund for Gastrointestinal Cancer Research, Pappas Family Research Fund for Pancreatic Cancer, NIH R01 CA124908, and NIH P50 CA127003 to CSF; NIH R35 CA197735 to S0; and from Department of Defense CA130288, Lustgarten Foundation, NIH/NCI K07 CA140790, the Noble Effort Fund, the Peter R. Leavitt Family Fund and Promises for Purple to BMW.

Competing interests None declared.

Patient consent Obtained.

Ethics approval Human Research Committee at the Brigham and Women's Hospital.

Provenance and peer review Not commissioned; externally peer reviewed.

\section{REFERENCES}

1 Siegel RL, Miller KD, Jemal A. Cancer statistics, 2016. CA Cancer J Clin 2016;66:7-30.

2 Vincent $A$, Herman J, Schulick $R$, et al. Pancreatic cancer. Lancet 2011;378:607-20.

3 Lennon AM, Wolfgang $\mathrm{CL}$, Canto Ml, et al. The early detection of pancreatic cancer: what will it take to diagnose and treat curable pancreatic neoplasia? Cancer Res 2014;74:3381-9.

4 Klein AP, Lindström S, Mendelsohn JB, et al. An absolute risk model to identify individuals at elevated risk for pancreatic cancer in the general population. PLOS ONE 2013;8:e72311.

5 Stewart SA, Weinberg RA. Telomeres: cancer to human aging. Annu Rev Cell Dev Biol 2006;22:531-57.

6 Slagboom PE, Droog S, Boomsma DI. Genetic determination of telomere size in humans: a twin study of three age groups. Am J Hum Genet 1994;55:876-82.

7 Valdes AM, Andrew T, Gardner JP, et al. Obesity, cigarette smoking, and telomere length in women. Lancet 2005;366:662-4.

8 Hezel AF, Kimmelman AC, Stanger BZ, et al. Genetics and biology of pancreatic ductal adenocarcinoma. Genes Dev 2006;20:1218-49.

9 van Heek NT, Meeker AK, Kern SE, et al. Telomere shortening is nearly universal in pancreatic intraepithelial neoplasia. Am J Pathol 2002;161:1541-7.

10 Wolpin BM, Rizzato C, Kraft P, et al. Genome-wide association study identifies multiple susceptibility loci for pancreatic cancer. Nat Genet 2014;46:994-1000.

11 Petersen GM, Amundadottir L, Fuchs CS, et al. A genome-wide association study identifies pancreatic cancer susceptibility loci on chromosomes 13q22.1, 1q32.1 and 5p15.33. Nat Genet 2010;42:224-8.

12 Rafnar T, Sulem P, Stacey SN, et al. Sequence variants at the TERT-CLPTM1L locus associate with many cancer types. Nat Genet 2009;41:221-7.

13 Mocellin S, Verdi D, Pooley KA, et al. Telomerase reverse transcriptase locus polymorphisms and cancer risk: a field synopsis and meta-analysis. J Nat/ Cancer Inst 2012;104:840-54

14 Campa D, Mergarten B, De Vivo I, et al. Leukocyte telomere length in relation to pancreatic cancer risk: a prospective study. Cancer Epidemiol Biomarkers Prev 2014;23:2447-54.

15 Lynch SM, Major JM, Cawthon R, et al. A prospective analysis of telomere length and pancreatic cancer in the alpha-tocopherol beta-carotene cancer (ATBC) prevention study. Int J Cancer 2013;133:2672-80.

16 Diez Roux AV, Ranjit N, Jenny NS, et al. Race/ethnicity and telomere length in the multi-ethnic study of atherosclerosis. Aging Cell 2009;8:251-7.

17 Hunt SC, Chen W, Gardner JP, et al. Leukocyte telomeres are longer in African Americans than in whites: The National Heart, Lung, and Blood Institute Family Heart Study and the Bogalusa Heart Study. Aging Cell 2008;7:451-8.

18 Wang $\mathrm{H}$, Chen $\mathrm{H}$, Gao X, et al. Telomere length and risk of Parkinson's disease. Mov Disord 2008;23:302-5.

19 Cawthon RM. Telomere measurement by quantitative PCR. Nucleic Acids Res 2002:30:e47.

20 McKay JD, Hung RJ, Gaborieau V, et al. Lung cancer susceptibility locus at 5p15.33. Nat Genet 2008:40:1404-6.

21 Shete S, Hosking FJ, Robertson LB, et al. Genome-wide association study identifies five susceptibility loci for glioma. Nat Genet 2009;41:899-904.

22 Amundadottir L, Kraft P, Stolzenberg-Solomon RZ, et al. Genome-wide association study identifies variants in the ABO locus associated with susceptibility to pancreatic cancer. Nat Genet 2009:41:986-90.

23 Wang $Z$, Zhu B, Zhang $M$, et al. Imputation and subset-based association analysis across different cancer types identifies multiple independent risk loci in the TERT-CLPTM1L region on chromosome 5p15.33. Hum Mol Genet 2014;23:6616-33.

24 Skinner HG, Gangnon RE, Litzelman K, et al. Telomere length and pancreatic cancer: a case-control study. Cancer Epidemiol Biomarkers Prev 2012;21:2095-100.

25 Durrleman S, Simon R. Flexible regression models with cubic splines. Stat Med 1989;8:551-61.

26 Bojesen SE, Pooley KA, Johnatty SE, et al. Multiple independent variants at the TERT locus are associated with telomere length and risks of breast and ovarian cancer. Nat Genet 2013;45:371-84, 84e1-2.

27 Kote-Jarai Z, Saunders EJ, Leongamornlert DA, et al. Fine-mapping identifies multiple prostate cancer risk loci at 5p15, one of which associates with TERT expression. Hum Mol Genet 2013;22:2520-8.

28 Campa D, Rizzato C, Stolzenberg-Solomon R, et al. TERT gene harbors multiple variants associated with pancreatic cancer susceptibility. Int $J$ Cancer 2015; 137:2175-83.

29 Willeit $\mathrm{P}$, Willeit J, Mayr A, et al. Telomere length and risk of incident cancer and cancer mortality. JAMA 2010;304:69-75.

$30 \mathrm{Ma} \mathrm{H}$, Zhou Z, Wei $\mathrm{S}$, et al. Shortened telomere length is associated with increased risk of cancer: a meta-analysis. PLOS ONE 2011;6:e20466.

31 Wentzensen IM, Mirabello L, Pfeiffer RM, et al. The association of telomere length and cancer: a meta-analysis. Cancer Epidemiol Biomarkers Prev 2011;20:1238-50.

32 Weischer M, Nordestgaard BG, Cawthon RM, et al. Short telomere length, cancer survival, and cancer risk in 47102 individuals. J Natl Cancer Inst 2013;105:459-68. 
33 Zhang $C$, Chen $X$, Li L, et al. The Association between telomere length and cancer prognosis: evidence from a meta-analysis. PLoS One 2015;10:e0133174.

34 Savage SA, Gadalla SM, Chanock SJ. The long and short of telomeres and cancer association studies. J Natl Cancer Inst 2013:105:448-9.

35 Hou L, Joyce BT, Gao T, et al. Blood telomere length attrition and cancer development in the normative aging study cohort. EBioMedicine 2015;2:591-6.

36 Benitez-Buelga C, Sanchez-Barroso L, Gallardo M, et al. Impact of chemotherapy on telomere length in sporadic and familial breast cancer patients. Breast Cancer Res Treat 2015;149:385-94.

37 Martin-Ruiz CM, Baird D, Roger L, et al. Reproducibility of telomere length assessment: an international collaborative study. Int J Epidemio/ 2015;44: $1673-83$.

38 Iles MM, Bishop DT, Taylor JC, et al. The effect on melanoma risk of genes previously associated with telomere length. I Nat/ Cancer Inst 2014;106.

39 Rode L, Nordestgaard BG, Bojesen SE. Peripheral blood leukocyte telomere length and mortality among 64,637 individuals from the general population. J Nat/ Cancer Inst 2015;107:djv074.

40 Zhang $\mathrm{C}$, Doherty JA, Burgess $\mathrm{S}$, et al. Genetic determinants of telomere length and risk of common cancers: a Mendelian randomization study. Hum Mol Genet 2015;24:5356-66.
41 Machiela MJ, Lan Q, Slager SL, et al. Genetically predicted longer telomere length is associated with increased risk of B-cell lymphoma subtypes. Hum Mol Genet 2016;25:1663-76.

42 Matsuda Y, Ishiwata T, Izumiyama-Shimomura N, et al. Gradual telomere shortening and increasing chromosomal instability among PanIN grades and normal ductal epithelia with and without cancer in the pancreas. PLOS ONE 2015;10:e0117575.

43 Daniali L, Benetos A, Susser E, et al. Telomeres shorten at equivalent rates in somatic tissues of adults. Nat Commun 2013;4:1597.

44 Walsh KM, Codd V, Smirnov IV, et al. Variants near TERT and TERC influencing telomere length are associated with high-grade glioma risk. Nat Genet 2014:46:731-5.

45 Codd V, Nelson CP, Albrecht E, et al. Identification of seven loci affecting mean telomere length and their association with disease. Nat Genet 2013;45:422-7, $7 \mathrm{e} 1-2$.

46 Youngren $\mathrm{K}$, Jeanclos $\mathrm{E}$, Aviv $\mathrm{H}$, et al. Synchrony in telomere length of the human fetus. Hum Genet 1998;102:640-3.

47 Kimura M, Gazitt Y, Cao X, et al. Synchrony of telomere length among hematopoietic cells. Exp Hematol 2010;38:854-9.

48 Okuda K, Bardeguez A, Gardner JP, et al. Telomere length in the newborn. Pediatr Res 2002;52:377-81. 